\title{
DE CODICIBUS QUAESTIONUM
}

Alexandri Quaestiones edidi ad normam eius codicis, quo uno nobis servatae sunt, Veneti Marciani 258. de quo copiose egi in praefatione V voluminis prioris p. $\mathrm{Vsq}$. nune haec et repeto et addo.

Membranaceus est saeculi decimi, foliis constat 324 formae minoris, hoc ordine Quaestionum libros continet: fol. $1-36^{\text {v }}$ librum vulgati ordinis quartum, 37r $-69^{\mathrm{r}}$ librum primum, $261^{\mathrm{r}}-290^{\mathrm{v}}$ secundum, $291^{\mathrm{r}--324^{\mathrm{v}}}$ tertium. intermediam partem De anima libri duo qui feruntur et De fato commentatio occupant. quarti autem vulgati ordinis libri titulus est in

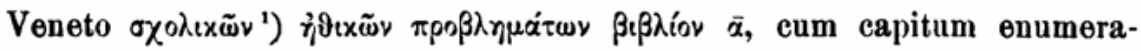
tioni verba praeposita sint $\sigma \chi 0 \lambda \iota x \tilde{\omega} \nu$ iterata in calce libri, cui praeterea littera $\mathrm{Z}$ subscripta est, quae quid

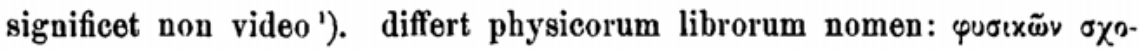

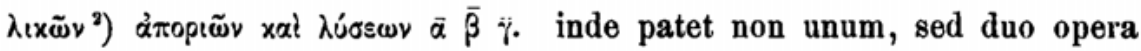
Quaestionibus contineri, quorum prioris praeter primum ceteri periere libri, alterius tres extant. servaverunt pristinam hanc distinctionem soli Marciani 258 et 261 . reliqui eo utuntur ordine, quo Victor Trincavellus, princeps editor, libros disposuit.

Eadem diligentia, qua ex vetusto exemplo De anima librum cum $\mathrm{V}^{1}$ mantissa descripsit librarius codicis V, usus est in Quaestionibus. mino-

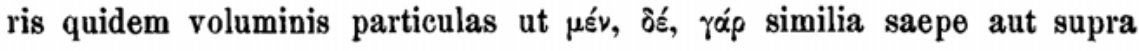
aut infra versum addidit, sed raro inveniuntur voces, quas in textu omissas in margine debebat supplere, of. 123,27 128,1 131,9 140,13 146,3

1) $Z(\eta \tau \eta \tau \tau$ (tov) supplendum esse suspicatur Diels, i. e. quaerendi sunt libri morales qui post primum desunt. similiter hoc signum in lacunis vel mendis a librariis interdum adhiberi notum est.

2) $\sigma$ o $\lambda(\omega v$ ante secundum et tertium librum. 
159,23. rarius tota enuntiata margini adscripsit, ut 83,23 100,27 130,15. saepius peccavit in fine versuum ita ut sive litteras sive syllabas omitteret: $5,24 \quad 9,19 \quad 15,28 \quad 37,9 \quad 89,1 \quad 99,24 \quad 108,33 \quad 119,9 \quad 122,12 \quad 125,11 \quad 129,22$ 130,17. 32 134,25. verborum ordinem immutavit 50,19 115,7 129,20 et saepe eum correxisse id quod primo seripserat magna liturarum copia indicat, quas omnes ne minoribus quidem neglectis adnotavi. raro autem priorem lectionem licet detegere, ut 7,4.9. $23 \quad 9,6 \quad 17,10 \quad 25,6 \quad 158,28$. contra fere desunt in Quaestionibus - id quod in libris De anima et De fato saepe animadvertimus - vestigia librarii de lectione describenda ita dubitantis, ut singulis litteris singulas alias supraseriberet (sed cf. 102,22). varias lectiones ex archetypo bis descripsit 7,10 et 54,8 ; item ex illo fluxerunt, quae addita sunt 142,7 et 144,34 .

v. c. V Venio ad veterem correctorem, quem dixi (I p. IX) totum codicem cum prototypo contulisse et fortasse non diversum esse a primo librario. cuius etiam in quaestionibus non desideratur cura et industria. nam voculas omissas hic supplevit $2,29131,31$ syllabas addidit 134,25 litteras et omissas adscripsit $71,975,33 \quad 128,19$ et corruptas correxit $63,1372,28$ $102,12131,30134,13$, interdum male servato archetypo deceptus est 102,20 et 29 . sed haec minora sunt prae magno et gratissimo numero punctorum, quae ab eodem adposita esse et atramentum testatur et calami gracilitas et summa cautio atque modestia, qua usus est huius recensionis auctor alienissima a posterioris correctoris $\left(\mathrm{V}^{2}\right)$ audacia, qui non tacite monuit, sed confidenter ea, quae displicuerant, mutavit. sunt autem tenuissima haec puncta aut supraposita singulis litteris aut in calce adpicta maxima ex parte eis, quae delendae videbantur. sic iure notantur e. gr. 48,1. $649,10.28$ 81,28 129,1 130,15 159,24. deinde adhaerent sive litteris sive syllabis non quidem delendis, sed corruptis vel

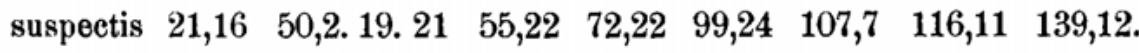
aperte autem collatorem, non criticum adgnoscimus e. gr. 160,2, ubi $\mu \varepsilon \gamma \alpha \lambda \circ \iota$ ou $\tau \varepsilon$ dederat prima manus, quod postea $\mathrm{m}^{2}$ optime mutavit in $\mu \varepsilon \tau \grave{\alpha} \lambda \hat{\sigma o ́}_{\rho} \tau \varepsilon$. haec etsi non perspexit vetus corrector vidit tamen id quod $\mathrm{m}^{\prime}$ scripserat non prorsus consentire cum archetypi obscura lectione et errorem in ot et $\tau \varepsilon$ inesse recte monuit: $\mu \varepsilon \gamma \alpha \lambda \dot{\partial} \iota$ o $\partial \dot{\tau} \varepsilon$. eodem modo corruptelam indicavit $51,2573,29$, emendandi periculum non suscepit. de accentu ita dubitasse videtur 40,1 41,25 42,15 120,24. causa latet 51,28. Archetypus Prototypi igitur videmus eam fuisse condicionem quae describentes variis difficultatibus vexaret. accedit quod accentibus et aspirationis indicatione caruit. quae Veneti librarius cum de suo arbi- 
trio addere deberet, saepe haesit; quo factum est, ut eae potissimum voces, quae diversa prosodia in varias notiones discedunt, ut $\ddot{\eta} \tilde{\eta}_{\hat{\eta}} \hat{\eta} \tilde{\eta}_{i}$

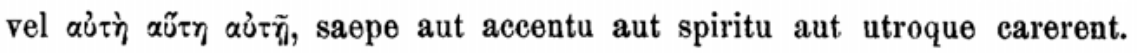
pauca delibabo ex multis $14,36 \quad 19,29 \quad 21,23 \quad 29,28 \quad 30,15 \quad 35,7 \quad 37,12 \quad 41,28$ 48,6 50,4 52,25 55,25 92,10 98,5 110,22 etc.

Deinde autem, id quod gravius est, saepenumero eis sive verbis sive verborum coniunctionibus spiritus et accentus deesse voluit, quae aut non intellexit aut corrupta esse plerumque recte suspicatus est. adponam nonnulla exempla

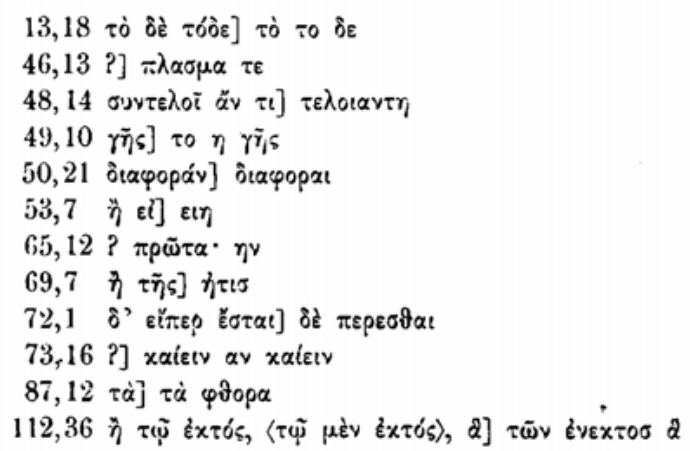

Ingratum editori talia officium impingunt, ut omnes has omissiones tradat, haud nescius multas casui non consilio deberi. graviora igitur in adnotationem recepi, quam ne nimis onerarem, reliqua in Additamento priore collegi.

Deinde continua scriptura vetustum exemplar scriptum fuit. quod diversa vitiorum genera docent, quae omnia hane tantum explicationem admittunt. iam pridem restituta sunt haec oủxaốva

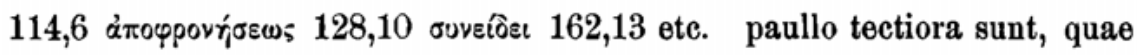

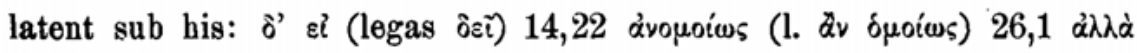

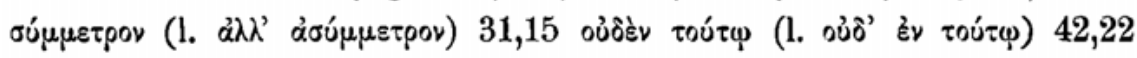

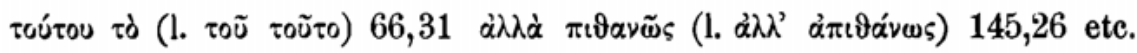
saepius perperam disereta novam labem traxerunt, cuius generis mendorum satis magna copia nune remota est, sed restare puto quae manum emendatricem expectent. certe de origine corruptelae non dubitabitur:

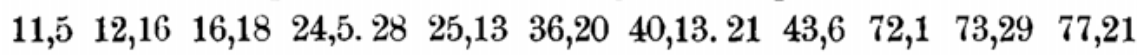
81,13. 26 85,32 98,1 103,22 105,3 107,37 145,33.

Uncialibus porro litteris exaratus fuisse videtur ille liber. quod

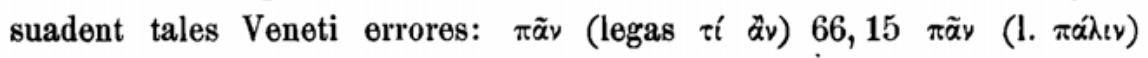

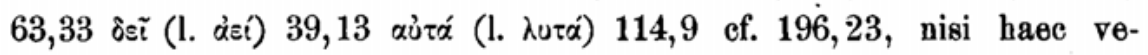


tustiora sunt. hoc autem certum est, si uncialibus scriptus erat codex, ex quo fluxit Venetus, multa compendia intermixta fuisse, cuius scripturae generis imaginem nobis praebent in Veneto et capitum rubra et marginales adnotationes et argumenti conspectus primo physico libro et morali praefixus (nam pinax qui ante alterum et tertium physicorum legitur minuseulis conscriptus est). tantus est numerus depravatarum syllabarum terminantium; ef. $7,2.9$ 8,18 10,23. 24 12,17.23. 27 15,19. 22 17,12 21,27 22,24 26,6 27,18. 28 29,5 $31,5.10 .20 \quad 36,25 \quad 39,17$ $48,370,28$ 83,4 112,3 etc. nolo hoc loco omnes Veneti corruptelas in sua genera distribuere. sed conducet duo breviter notasse, quae prae ceteris respicere debeat emendaturus. latissime enim patet vocalium inter se et diphthongorum permutatio, quae non solum in st et t, at et $\varepsilon$ offendit, sed tanta est, ut cuiusvis vocalis cum unaquaque confusae baud difficile sit exempla deprehendere. deinde iterum miserrima omissionum copia conquerenda est, de qua dixi in praefatione vol. I, p.X. sed taedet singillatim referre et litteras et syllabas et voces et enuntiata quae excidere. legentibus enim ultro se obtrudet lacera archetypi species instar cribri perforati. raro contra removendae sunt otiosae litterae syllabaeve, ut $25,1327,14.1535,2566,7 \quad 77,22129,1$; et casu videntur olim e margine in textum irrepsisse pauca illa, quae recidere debui 23,24 26,20-22 48,4-5 67,22 sq. 92,32.

$\mathrm{V}^{2} \quad$ Venio nunc ad id genus lectionum, quod in libris de anima $\mathrm{V}^{2}$ signavi. nam etiam Quaestiones doctus ille licet non eadem ubique industria pertractavit. permulta enim cum adnotaverit primo physico libro et moralibus problematis, rarescunt eius vestigia inde ab octavo capite libri alterius physici. confidentius autem nune postquam accuratissime iterum codicem examinavi eidem tempori has lectiones deberi certum esse contendo, ab eodem homine provenire verisimile. certe nemo poterit diversis auctoribus sua reddere. nam litterarum primo obtutu prorsus similium discrepantiae (quas in litteris $\delta$ et $\sigma$ esse non nego) tales sunt, quales facile homo doctus festinanter scribens admittat. neque ex atramento hic fusciore illic atriore aliquid concludere licet. immo unum ubique adhibitum esse eae voces arguunt, in quibus eadem coloris diversitas apparet. - neque de auctoritate earum nune aliter iudico. a viro provenerunt peritissimo, qui utinam omnes Alexandri libros pari cura pertractasset. eam enim operam praestitit scriptis per saecula neglectis, quam et postulamus et laudamus in primo editore. nam restituit hic litteras corruptas, prosodiam adpinxit, falso secreta recte distiuxit, otiosa 
rescidit, omissa supplevit, comparando ea, quae bis leguntur, recensuit, felici saepe acumine etiam e corruptioribus locis pristinum sensum elicuit. ita plus centies in uno libro primo textum sanavit. noli autem id quod iudicii sanitati contigit subsidiis tribuere nobis ignotis. Vetant hoc loci fere sexaginta eiusdem primi libri, ubi critico res minus prospere successit. dubitantibus pauca expromam, quae sana quoque eum corru-

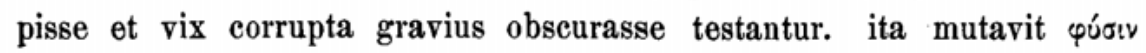

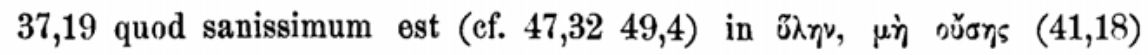

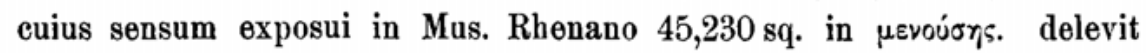

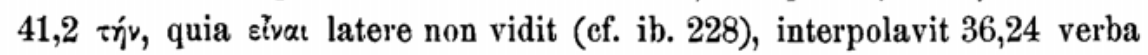

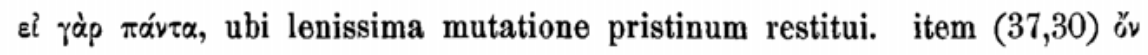

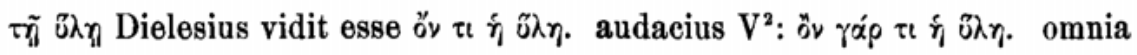
sana esse 36,2 modo pro Evì scripseris \&ิv el docuit Schwartz. ille Evit mutavit in Ėıvoía (nam in apparatu excidit $\mathrm{V}^{2}$ ante $\mathrm{B}^{1}$ quod corrigas).

Quare correcturas huius manus omnes accurate et adnotavi et pensitavi, sed sprevi ubi displicuere. neque eam secutus sum - nisi alia

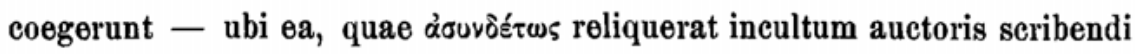
genus, copulavit.

Venetum bis ipse contuli 1881 et 1887 . ubi nil adnotavi huius codicis lectionem confidas in textu impressam esse.

Prater hune sex mihi innotuerunt apographi codices Quaestiones continentes: duo Veneti, Estensis, Neapolitanus, Vindobonensis, Vaticanus.

Venetum 261 descripsi vol. I p. $\mathrm{X}$ et ex codice $\mathrm{V}$ eum pendere docui $\mathrm{B}$ duobus locis 112,6 et 26, ubi evanidam Veneti $V$ lectionem is qui B confecit omisit simul fenestra indicans aliquid deesse. addo 48,14 quo loco

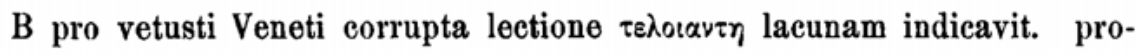
pius hic abest a $\mathrm{V}$ quam reliqui, nam solus veterem moralis libri inseriptionem et multas Veneti $\mathrm{V}$ lectiones servavit a reliquis mutatas, e. gr.

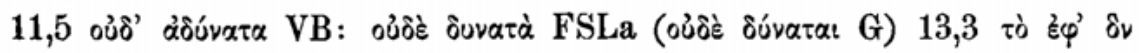

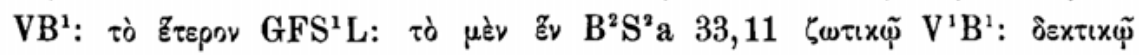

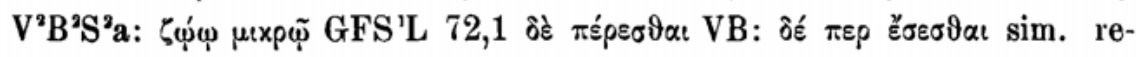

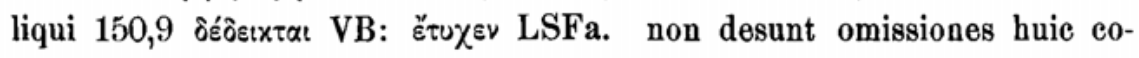
dici propriae 31,24 162,15. 16 . permirum autem est in tanta cum V similitudine id quod adnotavi ad 55,14 et 15, unde colligere oportet non descriptum esse ex ipso $\mathrm{V}$ codicem $\mathrm{B}$, sed intermedium esse inter ambos eum librum, cuius apographum est B. ille vero ad verbum consentiens cum $\mathrm{V}$ rara docti cuiusdam tentamenta passus est, quorum vestigium 55,14 in $B$ transiit. 
Alia via ex Veneto 258 descripti sunt Mutinensis Estensis III G 6 cod. chartaceus foliorum 400 (ut adnotatum est in p. 1) numeris non signatorum, saec. XV-XVI scriptus; continet post Alexandri commentarium in Aristotelis De sensu et sensili et Procli elementa physica Quaestiones et librum De fato. sequuntur Ps. Alexandri aliorumque problemata varia.

F Neapolitanus III D 12 chartaceus fol. 109 absolutus est die 27. mensis Junii 1523, ut testatur manus librarii Angeli Constantini. praeter Quaestiones nihil continet. ef. Cyrillus II, 384.

S Venetus Marcianus append. A. IV, cod. X chartaceus fol. 141 saec. XVI-XVII continet ante Galeni definitiones medicas Alexandri

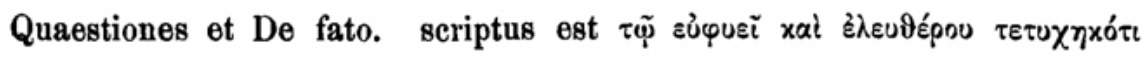

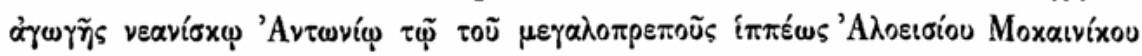
(Mocenigo).

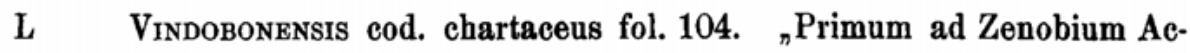
ciaiolum Florentinum ordinis praedicatorum die 14 Ianuarii 1497, postea autem ad Ioannem Sambucum 1536, ut ipsi propria manu ibi testantur, olim pertinuit" ef. Nessel IV, 18.

(G passim, FS magna ex parte ipse contuli, L totum contulit Mekler Vindobonensis.)

Communia sunt his libris haec: in omnibus problematum inseriptio ante moralem librum periit. deinde in tertio libro post oũ

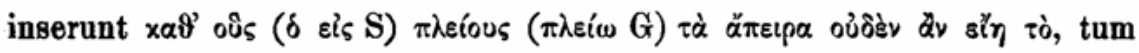
iterant verba $\dot{\omega} s$ pépos usque ad aǐtıov, quae 102,11-30 suo loco legebantur. animadvertendum autem verba contra sensum iterata eis mendis

$F^{a} \mathrm{G}^{\mathrm{a}} \mathrm{S}^{\mathrm{a}} \mathrm{L}^{\mathrm{a}}$ carere quibus inquinata sunt eadem, ubi iusta sede extant (ef. 102,20. 21. 23). itaque casu in eum codicem, ex quo descripti sunt GFSL, irrepsit folium ex alio codice, qui Venetum V cum maiore fide expressit. porro communis eis est magna in quarto libro lacuna, qua periere integra capita 5-8, quartum inde a verbis $\tau \tilde{\varphi} \pi \lambda \circ u ́ \tau \varphi(123,17)$, nonum usque ad verba זpocipoúnevor $(129,23)$. accedunt minores omissiones, ex.

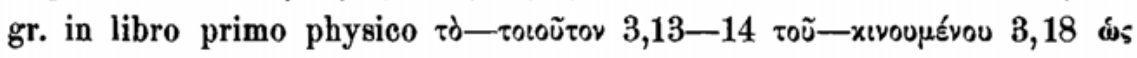

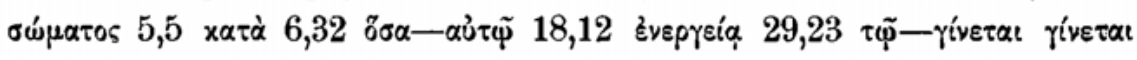

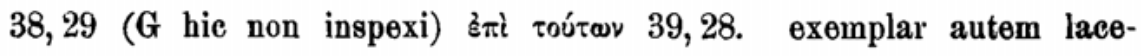
rum, ex quo fluxere, pro virili parte quisque ultro foedavit. exactam tabulam non desiderabis, sed scias nulli deesse suas lacunas, velut in F numeravi libri primi hiatus 12 maioris ambitus. accedunt corruptelae his quattuor peculiares, ef. ex libro primo $3,185,26$ 9,10 23,7.21. 23. 30 


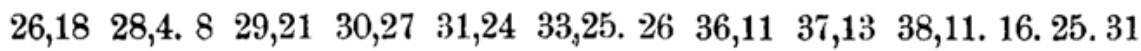
$39,2540,14.1941,2$ (ubique cum $V$ verum servavit $B$ ), nam neque ceterorum librorum menda colligam neque ea quae singuli suo Marte peccaverint.

Patrem autem codicum GFSL descriptum esse ex Veneto vel ex multis eorum lacunis cognoscitur. neque enim usquam hi libri opem ferunt, ubi in Veneto aliquid exidisse suspicamur, et 22,1 òv- $\omega s$ ideo omissum est in GFSL, quia hoc enuntiatum manus altera Veneti adscripsit ex comparatione sequentis capitis.

Deinde perlustres eos locos, quibus utpote corruptis in Veneto accentus et spiritus desunt. adponam nonnulla

$46,13 \pi \lambda \alpha \sigma \mu \alpha \quad \tau \varepsilon$ V: $\pi \lambda \tilde{\sigma} \sigma \mu \alpha \tau \varepsilon$ G: $\pi \lambda \dot{\alpha} \sigma \mu \alpha \tau \varepsilon$ SF : $\pi \lambda \dot{\alpha} \sigma \mu \alpha \tau \dot{L} \mathrm{~L}$

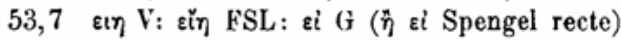

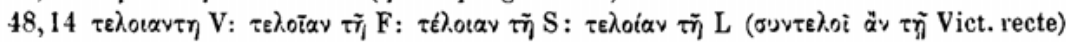

72,1 ò Aristoteles)

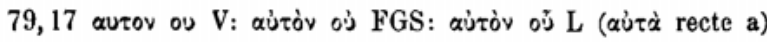

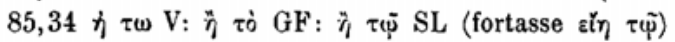

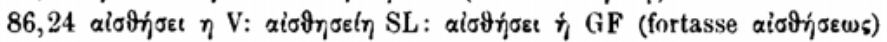

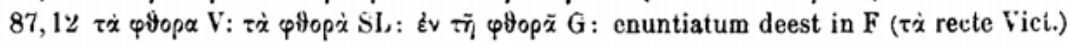

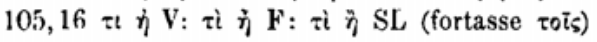

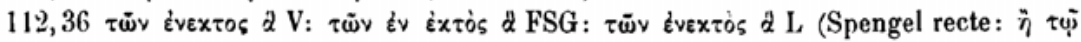

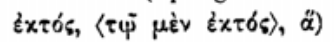

Vides quam incerti, ubi dux deest quem sequantur, titubent. idem docent vestigia veteris correctoris modo pressa modo neglecta. exemplo sunto

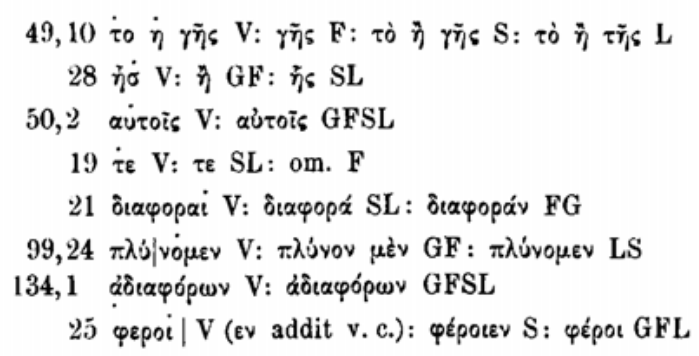

Secundae manus proposita aut sequuntur omnes (20,13. 25 24,4.5 $25,3027,928,11.30)$ aut neglegunt $(40,1 \quad 120,14$ 121,3.5) aut in diversas partes discedunt (e. gr. GLV': $\mathrm{SFV}^{1} 20,26-\mathrm{GV}^{\prime}$ : FSLV' 15,19 53,5 - GFSV': $\left.\mathrm{LV}^{1} 36,14\right)$. sed observare licet, ut B soleat alteri manui obsequi, ita cum prima malle ire FGLS. ex quibus consectarium est librorum GFSL archetypum secundam manum Veneti plerumque neglexisse, interdum secutum esse, haud raro autem et primam et 
alteram manum servasse. nam etiam veteris correctoris monita ex parte videtur ille adnotasse, quod ex apographorum dissensu consentaneum est.

Sed propero ad finem et paucos locos adscribam ex quibus, quae sit horum codicum affinitas, praecipue eluceat

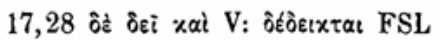

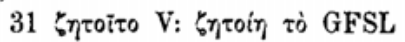

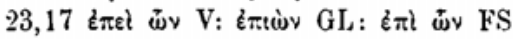

$26,8 \chi \rho \omega \mu$ et lacuna 10 litterarum $V^{1}: \chi_{\rho} \omega \mu\langle\varepsilon v \omega\rangle$ sive $\chi \rho \omega \mu\langle\varepsilon v \omega v\rangle V^{2}: \chi \rho \tilde{\mu} \mu \alpha \mu \dot{\varepsilon} \nu$

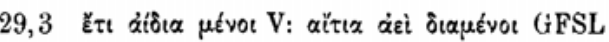
oùv GFSL

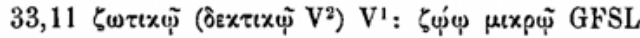

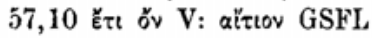

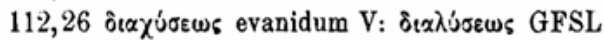

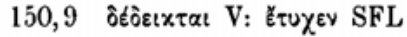

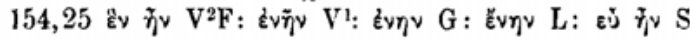

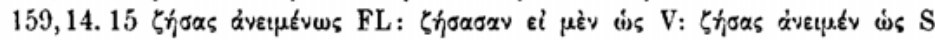

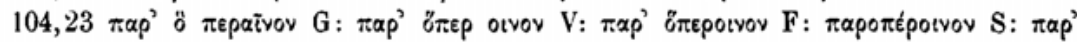

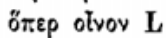

In extremis duobus locis medela inventa est et a FL et a G. quam provenisse puto ex margine eius libri, ex quo ducti sunt GFSL. nam hunc, postquam descriptus est ex Veneto, a docto homine correctum esse loci docent haud pauci, ubi GFSL a Veneti corruptelis liberi sunt. sunt autem in primo libro gravissimi: $4,235,33 \quad 6,29 \quad 7,2 \quad 24,17 \quad 27,23 \quad 38,9$ $39,1340,28.2941,15$ ef. $5,269,1031,24$. habes causam, cur non plane neglexerim libros pessimos, sed et in libri primi adnotationem multa receperim, et Vindobonensis collationem in Additamento altero proponam, ne desit integer huius classis testis.

K Vaticanus Urbinas 54 chartaceus saec. XVI continet fol. 1-105 Quaestiones, fol. $105^{\mathrm{v}}-136^{\mathrm{v}}$ librum De fato. accurate contuli librum De fato, quem vidi non quidem apographum esse, sed fratrem deteriorem libri anno 1536 impressi, qui ante librum De fato unicam usque ad annum 1842 continet Quaestionum editionem. cuius cum Vaticano eandem fore affinitatem suspicato mihi probavit et quod singularis inseriptio

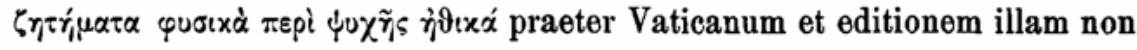
invenitur et quod collata quattuor primi libri capita prorsus consentiunt

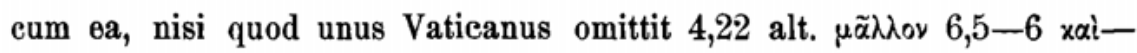

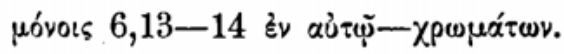

a Quare neglecto hoc libro vertam me ad editionem principem Trincavelli, quae cum dedicatoria epistola ad Petrum Danesium missa prodiit 
anno $1536^{1}$ ). continet autem recensionem Quaestionum ex pluribus codicibus conflatam, quos tamen omnes ex Veneto pendere probari potest.

Exeundum est a permultis locis, quibus a contra VB conspirat cum GFSL, e. gr. 3,26 4,2. 18. 23 5,2. 6 6,29 7,2 8,34 9,10 10,2. 4 12,12. 13

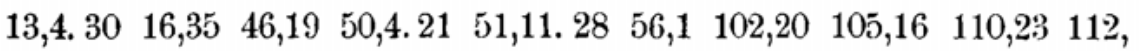
$26.36 \quad 130,16 \quad 133,28 \quad 137,16 \quad 139,2$.

Sed alia praeter hos libros suppeditabant auxilia Trincavello vel quicunque in eius usum hanc editionem paravit. nam neque iteratur III, 102,11 sq. et integer est moralis liber et maxima pars lacunarum, quibus laborant GFSL, resarta est in a (etsi non prorsus desunt, hiat enim a cum illis 153,4 et 162,17 ). accedit mira $55,14.15$ cum uno Veneío 261 similitudo. gravius autem hoc est. leguntur in duobus Venetis $B$ et $\mathrm{S}$ multae secundae manus correcturae valde memorabiles, $\mathrm{B}^{2} \mathrm{~S}{ }^{2} \mathrm{a}$ quarum maxima pars a Trincavello recepta est. ef. $3,15.18$ 7,30 $\begin{array}{lllllllllll}10,13 & 11,9 & 13,3 & 15,30 & 16,5 & \text { (हैं เ) } 17,32 & 18,13.16 .23 & 19,12.18 & 23,8 & 27,13\end{array}$ 29,19. 23 30,8.9 32,22. 29 33,1. 28 34,6. 7 36,3.8.12.25 38,23 39,13. 27 40,2.9.14. 21 41,28 etc. quas lectiones si quaerimus unde venerint in editionem principem, hoc certum est neque ex $\mathrm{B}$ neque ex $\mathrm{S}$ in eam migrasse. obstant enim primum aliae lectiones secundae Veneti 261 manus, quae neque in $\mathrm{S}$ neque in a inveniuntur $3,7.26 \quad 4,105,20 \quad 6,8.9 \quad 7,2 \quad 8,4$ 10,5. 24 11,5. $6.33 \quad 12,2.23 .25 \quad 18,4 \quad 19,29 \quad 25,8.13 \quad 33,7 \quad 42,25$ etc., deinde $S^{2}$ quoque sibi peculiares exhibet e. gr. 13,18 21,20 24,21 29,19, denique interdum et $S^{2}$ cum a conspirant contra $B(5,33 \quad 7,5 \quad 8,18$ 12,17 $48,3)$ et $B^{2}$ eum a contra $S(121,21)$.

Quare haec mihi videtur origo fuisse codicis a Trincavello impressi. adhibuit huius auctor primum exemplar familiae FSGL, gemellum ut videtur codicis S. suadent hoc multi loci, ubi Trincavellus cum uno S congruit e. g. 20,29 25,12 27,28 29,9 33,18. 19 53,22 etc. deinde consuluit Venetum 261, ex quo maximam partem supplevit earum lacunarum, quibus FSGL inquinantur, et redegit 55,14.15. sed accessit tertius codex, eui debentur eae lectiones, quae alteri manui codicum $B$ et $S$ cum a communes sunt. fuit is ipse quoque ex familia codicum FSGL, sed multifariam correctus ab homine docto saeculi $\mathrm{XV}$, cui neque ingenium deerat neque audacia qua utebantur haec tempora. quis fuerit nescimus, sed nolo tacere summis laudibus praedi-

1) "Venetiis in aedibus Bartholomaei Zanetti C'asterzagensis, aere vero et diligentia I. F. Trincaveli mense Aprili" cf. Add. 
cari a Trincavello Danesium, de quo haec ille: "tu mibi in Alexandri Quaestionibus quae ad calcem perductae ad editionem properant, eam operam et auxilium impertitus es, ut quidquid studiosi bonarum litterarum ex nostris laboribus se profecisse senserint, id vel maxime tibi debeant." ex hoc igitur libro non solum is, ex quo a impressa est, sed etiam codices $B$ et $\mathrm{S}$ correcti sunt. ${ }^{1}$ )

Explicatur autem hac ratione, cur tot lectiones classis FSGL secunda manu in Venetum B venerint. nimirum huius familiae librum vir doctus ille correxit ef. 4,2. $23 \quad 7,2 \quad 8,34 \quad 10,28 \quad 11,1.512,13 \quad 18,2020,14$. neque offendet, quod $\mathrm{S}^{2}$ multas lectiones alterius Veneti 258 manus in $\mathrm{S}$ invexit, quas cum fratribus hic praeterierat. nam non solum coniecit, sed etiam ex Veneto $B$ (quem scimus fere consentire cum $V^{2}$ ) passim inspecto suum librum correxit. ita factum est, ut saepe $\mathrm{S}^{2} \mathbf{a}$ consentiant

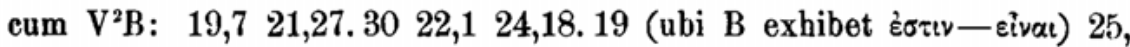
$22.2527,1731,932,533,1934,7$. simul causam videmus, cur 46,10 130,13 cum uno Veneto $B$ congruant $\mathrm{S}^{2} \mathbf{a}$.

Ingeniose dixi coniecisse auctorem lectionum $\mathrm{B}^{2} \mathrm{~S}^{2} \mathrm{a}$ signatarum. vel emendavit corrupta, e. gr. $11,9 \quad 15,30 \quad 16,5$ (气้זı) $17,32 \quad 18,13$. $16 \quad 30,9 \quad 36,12$. suspicio autem aliis praeter Venetum 258 fontibus eum usum esse quominus nascatur, haec tabula puto impediet.

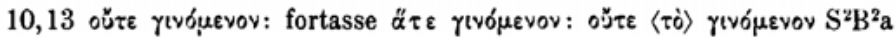

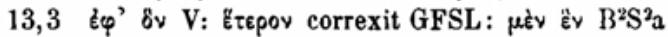

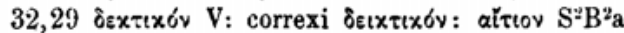

$34,12 \pi \varepsilon \sigma \varepsilon i \tau \alpha t \mathrm{~V}^{1}: \pi \dot{\varepsilon} \sigma \sigma \varepsilon t \tau \varepsilon$ correxit $\mathrm{V}^{2}: \varepsilon^{2} \times \pi \varepsilon \sigma \varepsilon i \tau \alpha t \mathrm{~S}^{2} \mathrm{a}$

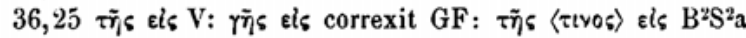

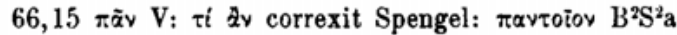

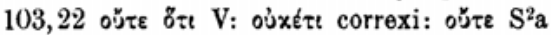

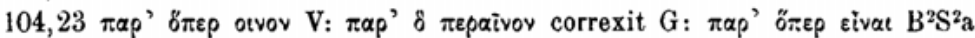

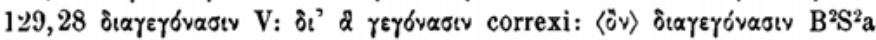

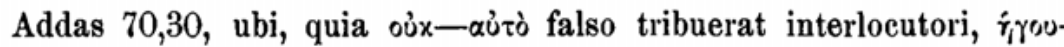

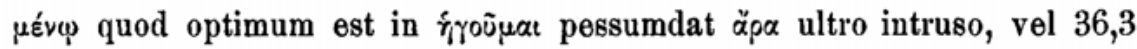
ubi videre licet quomodo interpolationi secundae manus Veneti 258 novam adiungant $\mathrm{B}^{2} \mathrm{~S}^{2} \mathrm{a}$. porro ef. $46,1.251,2 \quad 73,16 \quad 129,25$.

1) Difficile vero est de eis secundae manus lectionibus iudicare, quae aut $\mathrm{S}$ aut $\mathrm{B}$ peculiares sunt aut alterutri cum a communes. latet enim, utrum bic accesserint, an illic omissae sint. atque levidensia sunt, quae unus $\mathrm{S}^{2}$ praebet. tot autem et tam ingeniosae coniecturae, quae secundae Veneti B manui debentur, - etiam ad librum De fato in uno boc codice leguntur notatu dignissimae eiusdem manus adnotationes videntur ad huius codicis possessorem redire cum alienis sua inventa adnotantem. baec omnia diligenter collegi et in apparatu proposui. 
Non licebat hane de codicibus disputationem magis contrahere. Bagolinus sed oo brevius nune de Bafiolins (patris et filii) versione referam, quae primum ${ }^{1}$ ) prodiit Venetiis 1541 (editione anni 1559 usus sum). in adnotationem meam eam non recepi, quia confecta est ad exemplum talis libri, qualem modo descripsi. omnia enim additamenta adhibuit quae praebet $\mathrm{B}^{2} \mathrm{~S}^{2} \mathrm{a}$ et consentit cum $\mathrm{B} 55,14$. 15. hoc tantum addo ipsa editione Bagolinum non usum esse; verba saltem 136,3, quae a amisit, Bagolinus vertit.

Ab integris codicibus venio ad umbram codicis, quam retinuerunt lectio- Vict. nes, quas Monacensi exemplo editionis Venetae adscripsit Petrus Victorius, quas, cum ut ipse hic inspicerem hune librum LaUbmannı viri doctissimi benevolentia mihi contigisset, non ab una sed a diversis duabus manibus adpositas esse vidi. qua re inter vetustiorem manum eamque nitidiorem per omnes libros conspicuam, cui pleraeque lectiones debentur, distinxi et neglegentiorem paulloque recentiorem, cui interrogationis signum in adnotatione adposui. sed has ipsas lectiones, de quibus dubitaveram, certe Victorii esse nune scio, postquam benigne Rudolfus Schosll mea causa exemplar Monacense cum aliis Victorianis reliquiis comparavit. contra incertum esse me docet Schoell, utrum maior illa pars lectionum iussu Victorii a discipulo addita sit, an ab ipso Victorio priore tempore adscripta. sed hoc illo verisimilius esse. inveniri enim etiam hanc manum inter Victoriana, nam prorsus simili scriptura argumentum adnotatum esse margini Rhetoricorum ad Alexandrum.

Multas ex his lectionibus secutus sum, ut 13,26 24,9 28,10 29,22 48,6.

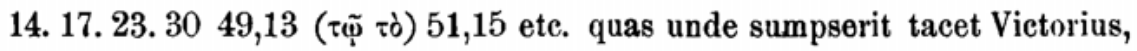

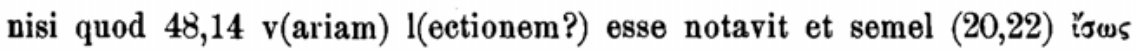
adscripsit, ex quo patet hane certe lectionem coniecturae deberi, non codicibus. alia addere possum aut falso aut inutiliter novata 6,1.15. 23 $12,1713,2519,1224,126,529,2131,640,146,24 \quad 48,2549,24$ etc. atque si quis ex 48,14 quo loco praeclare emendatur, quod in nostris codicibus corruptum est omnibus, concludere velit auxilia Victorio patuisse nobis negata, me non habebit adstipulantem. tanta enim est harum lectionum $\begin{array}{lllllll}\text { cum Veneto } 261 \text { cognatio (cf. 10,25 11,5 } & 12,15 & 15,17.22 & 18,4.29 & 22,9\end{array}$ $23,2527,14.1528,2336,840,14$ etc.), ut boc libro sive huius simili imprimis usum esse Victorium existimaverim, praeterea autem et suis aliorumque inventis et Aldino fortasse Aristotele (cf. 77,3 81,13 156,30. 33).

1) an 1516? Neutram editionem vidi, cf. Hoffmann Lex. bibl. p. 111. 
Sp. Victorianis primum usus est Srengelius, eui haec cum Trincavelli editione fundamentum fuere libri qui prodiit Monachii 1842. in quo multa editor emendavit, quae non potuit non expedire vir ut ille eximius. Sed plura expectamus a tali. nam pace viri doctissimi dictum sit festinationis, qua liber confectus sit, vestigia non prorsus latere.

Inter ExCERPTA manuscripta, quae mihi innotuerunt, nulla sunt, quae ad alium fontem redeant, quam librorum quos commemoravi. itaque quam brevissime de eis referam.

Sunt autem physicorum capita $1-16$ libri alterius excerpta in $\mathrm{H}_{\Lambda \mathrm{V}}$ NIENSI, de quo mox iterum disputabo. quae a Veneto $\mathrm{V}$ pendere arguit vel una corruptela 48,14. sed non ex ipso descripta sunt, etsi cum fide eum repraesentare solent. fortasse Veneti 261 simili libro usus est excerptor, quia 55,14. 15 inter manuscriptos Hauniensis unus cum B consentit. quem si exscripsit excerptor, praeterea alios libros usurpavit, nam lectionem 48,14 non potuit ex $B$ sumere. sunt autem etiam Hauniensi propria nonnulla, ut 47,25 48,7 49,11. 12.

R Codex PARISINus 1996 miscellaneus saec. XVI continet fol. 5-9. quaestionem I, 4 II, $4.5 \mathrm{III}, 13$. I, $4^{\text {1) }}$ contulit Immisch.

Codex Leydensis Scaligeri 51 saee. XV-XVI continet fol. 1-3 quaest. I, 4 (cf. Catal. Bibl. publ. 1716 Lugd. p. 341); contuli ipse. et Parisinum et Leydensem ex capite collato I, 4 patet ex Veneti 258 apographo descriptos esse, cuius fere omnes lectiones exhibent, ubi non lacunis deformati sunt, quibus uterque abundat (cf. 9,14, ubi legas póvx $\mathrm{VBRS}^{2}$ ) 10,23.24. 30 11,5,33 et cetera, quae ex $\mathrm{R}$ in adnotationem recepi, quocum semper consentit Leydensis. nam gemini sunt, quod etiam

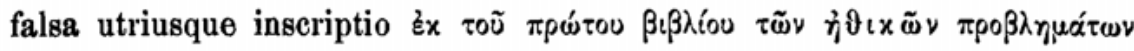
docet. ubi a Veneto dissentiunt, errores sunt. fructum ex neutro critici percipient.

N Moralis libri capita III, XXX, X excerpta leguntur in recentioribus nonnullis codicibus (Lavrentiano 85,1 Vaticanis 1622, 269 II Barberino II, 44 Mutinensi 197 (II G 4) Berolinensi Access. 1889 304²) commentarii Michaelis ad Aristotelis Ethica Nicomachea, fini libri quinti sine inscriptione

1) Idem exhibent excerptum Parisini 2077 et 2544, hic sub eodem titulo, atque Parisinus 1996 et Leydensis. non contuli. in catalogo librorum Parisinorum (anni 1740 p. II) praeter hos libros recensentur hi: solutiones quarundam difficultatum: 1268. 1630 quaestiones naturales 1883. 1884. 1893. 2047. 2047 A 2048. 2230. 2381. 3044. omnes continent diversa sequioris aetatis problemata, Alexandri quaestiones non insunt (Immisch). 
adiuncta. cuius rei notitiam accuratamque horum capitum cum quinque libris collationem comitati debeo Gustavi Heylbut, qui Mus. Rhen. 39,312 breviter rem exposuit. pendent autem ex eo libro, qui pater erat codd. FSGL. hoc docent cum lectiones 162,4.5.15.16 (ubi N consentit cum

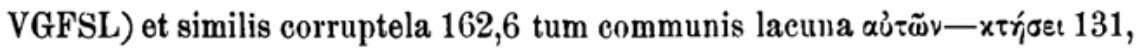
13 in $\mathrm{L}$ et $\mathrm{N}$. accedunt et omissiones et vitia his excerptis propria, ut

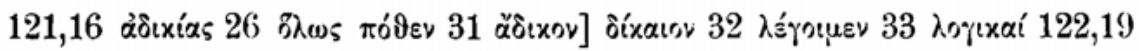

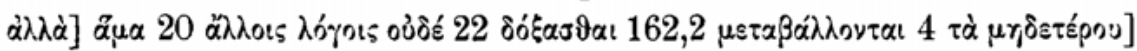

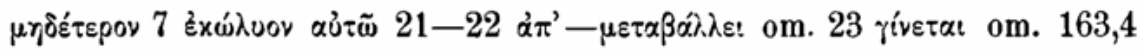

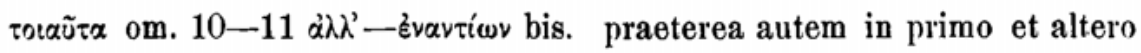

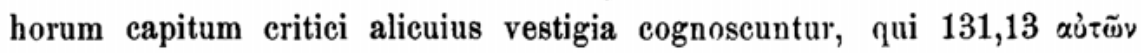

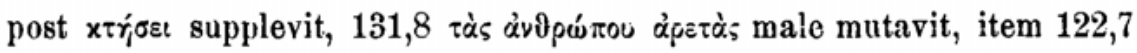

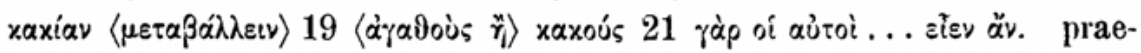
cipue autem finis huius capitis memoratu dignum est, ubi notes: 24 oútws

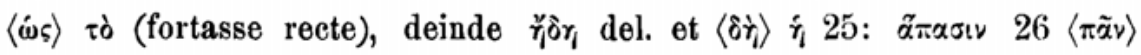

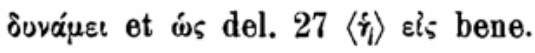

Vindobonensis CCCXI membranaceus in $4^{0}$ nitide scriptus cum va- M riis excerptis et philosophis et rhetoricis fol. $26^{\mathrm{r}}-48^{\mathrm{v}}$ septem moralis libri capita continet hoc ordine disposita: 25. 27. 29. 22. 24. 3. 10, quibus apud Nesselium (p. IV, p. 149) fictum nomen inditum est Alexandri ,liber de virtutibus'. excerpta sunt ex simili codice ac $\mathrm{N}$, quod lacuna docet 131,13. sed desunt tertio capiti in M coniecturae codicum N. levioribus exceptis consentit M cum L. contulit Mekler. 